\title{
Light emission from dye-doped cholesteric liquid crystals at oblique angles: Simulation and experiment
}

\author{
L. Penninck, ${ }^{*}$ J. Beeckman, P. De Visschere, and K. Neyts \\ ELIS (Electronics and Information Systems Department), Ghent University, St. Pietersnieuwstraat 41, Ghent, Belgium and \\ Center for Nano- and Bio-photonics, Ghent University, St. Pietersnieuwstraat 41, Ghent, Belgium
}

(Received 22 December 2011; published 9 April 2012)

\begin{abstract}
Dye-doped cholesteric liquid crystals with a helical pitch of the order of a wavelength have a strong effect on the fluorescence properties of dye molecules. This is a promising system for realizing tunable lasers at low cost. We apply a plane wave model to simulate the spontaneous emission from a layer of cholesteric liquid crystal. We simulate the spectral and angle dependence and the polarization of the emitted light as a function of the order parameter of the dye in the liquid crystal. Measurements of the angle dependent emission spectra and polarization are in good agreement with the simulations.
\end{abstract}

DOI: 10.1103/PhysRevE.85.041702

PACS number(s): 42.70.Df, 42.79.Kr, 78.20.Bh, 78.15.+e

\section{INTRODUCTION}

The self-ordering and easy tunability of liquid crystals have already led to an impressive number of applications. Recently, the use of liquid crystals has been suggested in many types of devices such as tunable filters [1], tunable optical chips [2], nonlinear optical devices, etc. In this article we focus on light emission from liquid crystal devices. In past years liquid crystal lasers have been developed with interesting properties that are difficult to achieve in other laser systems, most notably tunability and low fabrication cost [3].

Cholesteric liquid crystals (CLC), in which the director describes a helix with submicrometer pitch, are a class of materials with very interesting optical properties. The periodic structure of the CLC leads to a photonic band gap (PBG). Excited dye molecules inside the CLC no longer emit photons into the PBG region. When a sufficient number of dye molecules is excited, laser emission is observed at the edge of the band gap. Such lasers can be tuned by electrical [4], mechanical [5], or thermal [6] means. A more complete overview can be found in review papers $[7,8]$.

The performance of light emitting liquid crystal devices may be significantly improved by using more advanced optical structures. In order to develop new optical architectures a fundamental understanding of the emission process and accurate numerical design tools are necessary. The propagation of light waves in an infinite CLC has been extensively studied over the past decades. Analytical solutions for propagation along the helical axis were found by de Vries [9] and Kats [10]. For propagation under oblique angles several approximate solution methods were proposed [11] in the 1980s. About 10 years later an analytical solution was found [12]. Numerical studies [13] of finite structures were made with Berreman's $4 \times 4$ method [14].

The case of light propagation through a CLC is thus well understood. The problem of light emission from a CLC is more involved since the interaction between the emitting dipole and the electromagnetic modes and the finite dimensions of the structure have to be accounted for. The effects of the CLC on the fluorescence spectrum and polarization were first studied

*lieven.penninck@elis.ugent.be by Pollmann et al. [15] for wavelengths much shorter than the PBG (known as the Mauguin regime). Recent studies [16] have focused on emission wavelengths near the PBG (Bragg regime). An analytical model for the spontaneous emission from a CLC in the normal direction has been proposed by Schmidtke and Stille [17] based on the electric field profile of the eigenmodes of the CLC. This analytical approach is limited to basic structures and directions for which the eigenmodes are known. For more complex structures and/or emission directions for which the eigenmodes are not known, "brute force" numerical methods such as FDTD (finite-difference time domain) [18] can be used. However, such methods consume a lot of computer time and memory.

In this work we simulate the spontaneous emission from a one-dimensional (1D) CLC stack based on a decomposition of the field in plane polarized waves [19]. The advantage of this method is that complex stacks can be modeled with very limited computational effort. We have used this method to investigate the angle and polarization dependence of the emitted spectrum and the influence of the orientation of the emitting dye. The simulation results are compared to the measured spectrum for different polarizations and emission angles.

The materials and experimental methods are detailed in Sec. II. A simplified analytical model and the detailed numerical formulas are explained in Secs. III A and III B, respectively. In Sec. IV the experimental, theoretical, and numerical results are compared and discussed.

\section{EXPERIMENTAL PROCEDURE}

For the experiments we use a mixture of the nematic liquid crystal E7 (Merck) with a chiral dopant BDH1305 (Merck) and a laser dye DCM (Exciton). The three materials are mixed in a ratio of $94.03 \% / 5.07 \% / 0.9 \%$ by weight, respectively. To determine the molecular order parameter $S_{\text {dye }}$ of the dye a mixture containing no chiral dopant is made $(0.98 \% / 99.02 \%$ by weight of DCM/E7). The mixtures are heated above the clearing point and then returned to room temperature; the mixtures were stirred throughout this process.

Premade single pixel cells (Instec) are filled with the mixtures by capillary action. The cells consist of two parallel 


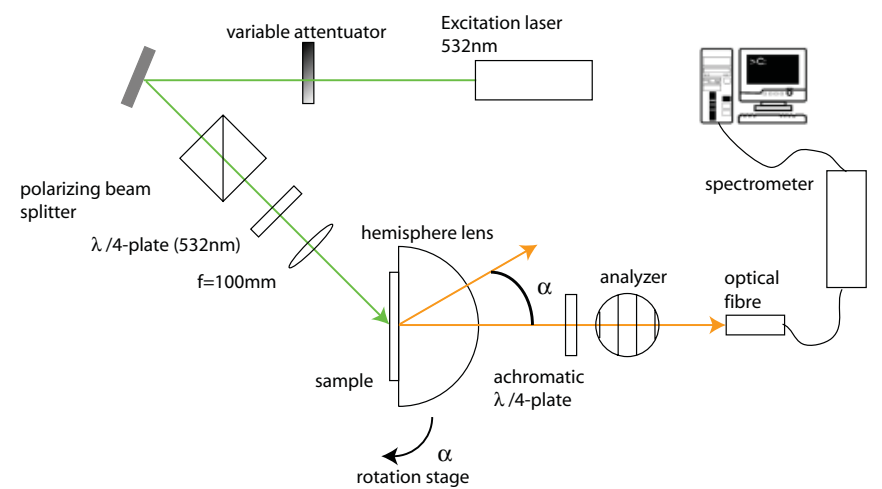

FIG. 1. (Color online) Spectral fluorescence measurement setup. The sample is excited by a circularly polarized laser beam. The emitted spectrum is detected by a spectrometer. The sample is placed on a rotating stage and optically coupled to a hemispherical lens.

glass plates, each covered with an indium tin oxide (ITO) electrode and a planar alignment layer. The glass plates are separated by $6.8 \mu \mathrm{m}$ spacers and the rubbing direction is antiparallel. The cells are filled at $70^{\circ} \mathrm{C}$ and are then slowly cooled to room temperature.

The transmission spectrum at normal incidence is measured using a transmission spectrophotometer (PerkinElmer Lambda 35). The emitted spectrum is measured using the setup depicted in Fig. 1. A cw frequency-doubled Nd:YAG laser $(\lambda=532 \mathrm{~nm})$ is used to excite the sample. The laser is passed through a variable attenuator to control the incident power and a polarizing beam splitter cube and quarter wave plate to ensure circular polarization of the excitation beam. The beam is then focused onto the sample by a lens. The sample is mounted onto a rotating stage to detect emission into different inclination angles $\alpha$. A glass hemisphere allows the measurement of light emitted under angles which are otherwise trapped in the glass plates by total internal reflection. The hemisphere is optically coupled to the sample using an index matching gel. This technique allows us to measure the emission into the glass substrate with minimal reflection at the glass-air boundary, since any ray coming from the center of the hemisphere impinges normally on the hemisphere surface. The emitted light is collected by an optical fiber and detected using a spectrometer (Qwave 390-950 nm). To measure the degree of left- and right-handed circular polarization, an achromatic quarter wave plate and rotatable analyzer are placed in the path of the emitted light. For the measurement of the degree of orientation of the dye in the nonchiral mixture, the quarter wave plate is omitted. The angle between excitation and detection is fixed at $135^{\circ}$, so the excitation beam is incident at $45^{\circ}$ for the measurement at $\alpha=0^{\circ}$.

\section{NUMERICAL AND ANALYTICAL MODELING}

The orientation of the liquid crystal director in a CLC with right-handedness is represented in Fig. 2. The liquid crystal molecules form a right-handed helical structure. Over a pitch distance $P$ the liquid crystal molecules have rotated a full $360^{\circ}$ around the helical axis.

The periodic variation of the director (pitch $P$ ) creates a periodic variation in the dielectric tensor with period $P / 2$. This one-dimensional variation generates a 1D photonic band gap (PBG) similar to a Bragg-type mirror. Inside the PBG the refractive index of one of the eigenmodes of propagation becomes imaginary (see, e.g., Ref. [20]), meaning this mode is evanescent. The CLC acts as a perfect reflector for that mode. In literature different conventions are used to describe circular polarization and care must be taken when comparing different references. In this work we assume that the electric field of a circularly polarized plane wave propagating in the $+z$ direction (to the right in Fig. 2) is

$$
\mathbf{E}(\omega, t)=\operatorname{Re}\left(\left[\begin{array}{c}
1 \\
\pm j \\
0
\end{array}\right] E \exp (j \omega t-j k z)\right) .
$$

The + sign describes circularly polarized light with righthandedness (top in Fig. 2), the electric field vector $\mathbf{E}$ rotates from $x$ to $y$ with increasing $z$ (describing a right-handed helix with increasing $z$ ). The - sign describes circularly polarized light with left-handedness (describing a left-handed helix with increasing $z$ ). If the LC director rotates with

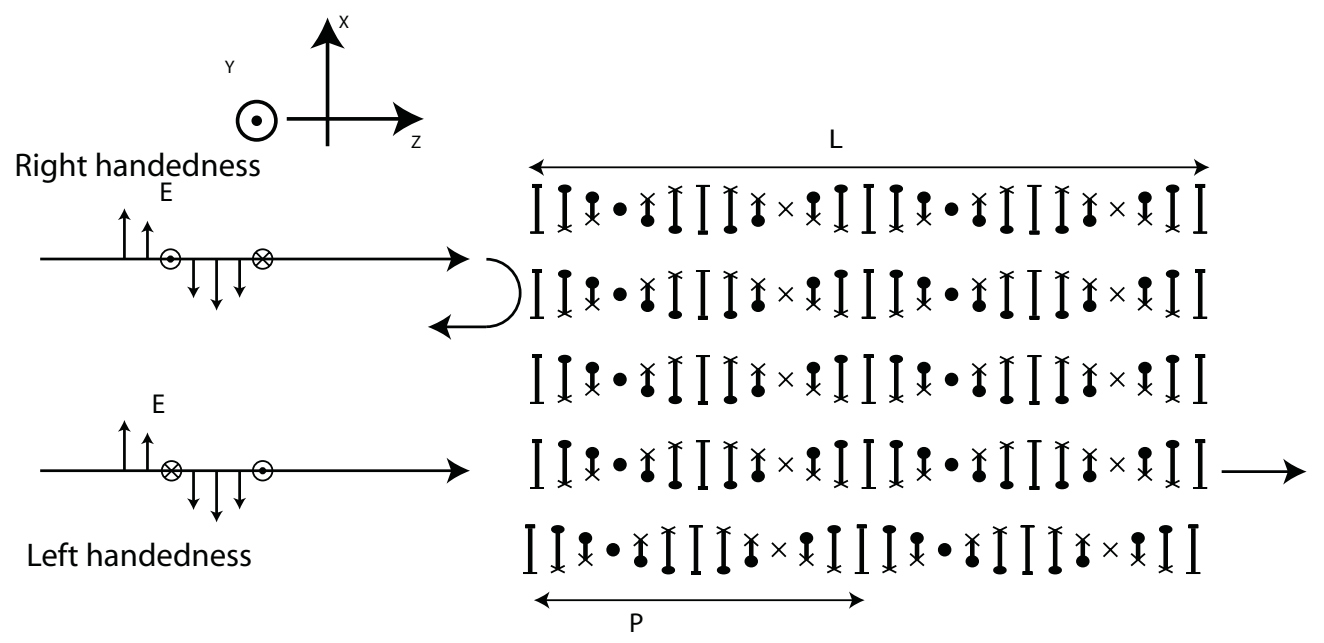

FIG. 2. Liquid crystal orientation in a right-handed CLC. The polarization with right-handedness (top) is reflected. The polarization with left-handedness (bottom) is transmitted. Dots and crosses are used to visualize the rotation sense of the LC director. 
right-handedness, then the polarization with right-handedness is reflected in a certain spectral region [21], the PBG. For an infinitely thick CLC, the reflection band in the perpendicular direction is defined by $n_{o} P<\lambda<n_{e} P$ where $n_{o}$ and $n_{e}$ are the ordinary and extraordinary refractive indices of the liquid crystal.

The PBG has a remarkable effect on the light emission from dye molecules inside the CLC. Because evanescent waves do not carry any power (in a loss free medium), no light can be emitted in the PBG. Another interpretation is that the density of states in the PBG is zero (at least in the perpendicular direction) and as a result, the excited dye molecule cannot decay via emission of a photon with wavelength and polarization in the PBG.

Just outside of the PBG, waves can be emitted. At the edge of the PBG the reflection coefficient oscillates rapidly with the wavelength. Here the emitted light will be subject to strong interference effects. When the reflections are in phase, a peak will occur in the emission. When reflections are in antiphase, a minimum will be seen. This can also be interpreted as an increase (decrease) in the optical density of states.

\section{A. Analytical solution for normal emission}

The spectrum emitted by a fluorescent dye perpendicular to the substrate can be calculated using the known eigenmodes of the CLC [17]. In this section we will derive a simple formula for the location of the peaks in the emission spectrum at both sides of the PBG, depending on the thickness $L$ of the CLC slab, for a dye molecule in the middle of the slab. The CLC is characterized by the pitch $P$ and the dielectric properties of the liquid crystal $\varepsilon_{\text {avg }}=\left(n_{e}^{2}+n_{o}^{2}\right) / 2$ and $\Delta \varepsilon=n_{e}^{2}-n_{o}^{2}$.

The amplitude of the electric field (in polarization state $i$ ) emitted by the dye is given by [19]

$$
E_{\mathrm{CLC}, i}^{r}=\frac{E_{\infty, i}^{r}+r_{i}^{l} E_{\infty, i}^{l}}{1-r_{i}^{l} r_{i}^{r}}
$$

assuming the two circular polarization states are not coupled by reflection on the CLC. This condition holds for the eigenmodes of polarization in the CLC. $r_{i}^{r / l}$ is the complex amplitude reflection coefficient (at the emission site) for a plane wave (amplitude $E_{i}^{r / l}$ ) traveling to the right or left in Fig. 2 with polarization $i$.

When $\left|1-r^{l} r^{r}\right|$ becomes zero a discrete, infinite peak will be seen in the emitted spectrum. When $\left|1-r^{l} r^{r}\right|$ becomes small a finite peak will appear. For the polarization where a PBG exists $r_{i}^{r / l}$ can be near unity and sharp peaks in the emission spectrum occur. For the polarization where no PBG exists, $r_{i}^{r / l}$ is near zero and the spectrum of a dye in an infinite medium will be emitted.

For the reflection coefficient $r$ for circularly polarized light normally incident on a CLC with pitch $P$, thickness $L / 2$, and with the same handedness as the CLC an approximate analytical expression can be found [21]:

$$
r=\frac{-j \kappa \sinh \left(s \frac{L}{2}\right)}{s \cosh \left(s \frac{L}{2}\right)+j \frac{\Delta k}{2} \sinh \left(s \frac{L}{2}\right)} .
$$

$\kappa, \Delta k$, and $s$ are shorthand notation for

$$
\begin{aligned}
\kappa & =\frac{\pi \Delta \varepsilon}{2 \lambda \sqrt{\varepsilon_{\text {avg }}}}, \quad \frac{\Delta k}{2}=\frac{2 \pi \sqrt{\varepsilon_{\text {avg }}}}{\lambda}-\frac{2 \pi}{P}, \\
s^{2} & =\kappa^{2}-\frac{\Delta k^{2}}{4} .
\end{aligned}
$$

The power reflection coefficient of an infinitely thick CLC is found by taking the limit of Eq. (3) for $L \rightarrow \infty$ :

$$
R=|r|^{2}=\left\{\begin{array}{ll}
1 & \text { for }-\kappa<\frac{\Delta k}{2}<\kappa \\
\frac{\kappa^{2} \sin ^{2}\left(|s| \frac{L}{2}\right)}{\frac{\Delta k^{2}}{4}-\kappa^{2} \cos ^{2}\left(|s| \frac{L}{2}\right)} & \text { for } \quad \frac{\Delta k^{2}}{4}>\kappa^{2}
\end{array} .\right.
$$

Inside the PBG (first condition) all power is reflected. Outside the PBG (second condition), the reflection coefficient varies as a sine squared. The distance between two maxima of reflection decreases with increasing thickness. The two edges of the PBG that can be found from the condition Eq. (5) are slightly different from the exact values $n_{o} P<\lambda<n_{e} P$. This deviation is due to the approximation $k=2 \pi \sqrt{\varepsilon_{\text {avg }}} / \lambda$ for the wave vector in the CLC in Eq. (4), which in fact only holds for very small $\Delta \varepsilon$.

For a dye molecule in the middle of the CLC structure the thickness is $L / 2$ in both directions and $r^{r}=r^{l}=r$. For the complex number $r^{2},\left|r^{2}\right| \leqslant 1$ and this amplitude varies much more slowly than the phase with changing wavelength. In this case, the quantity $\left|1-r^{2}\right|$ will reach a minimum close to the wavelengths where $r^{2}$ is a real and positive number, in other words near $\arg \left(r^{2}\right)=0$. After some algebra this leads to the following conditions for the peak wavelengths:

$$
|s| \frac{L}{2}=\frac{\pi}{2}+m \pi \text { and } \frac{\Delta k^{2}}{4}>\kappa^{2} .
$$

$\Delta k^{2} / 4>\kappa^{2}$ indicates that the peaks only occur outside the PBG. When $|s| L / 2=\pi / 2+m \pi, r^{2}$ becomes a real positive number. The condition for an emission peak in the spectrum for dye molecules that are not in the middle of the CLC can be found by calculating $r_{i}^{r / l}$ with Eq. (3) using two different thicknesses for $L$ and setting $\arg \left(r_{i}^{l} r_{i}^{r}\right)=0$.

\section{B. Plane wave expansion simulation}

The emission from a CLC in the normal direction can be modeled with analytical methods [17]. However, for more complicated optical designs (e.g., different director configurations or optical cavities) the analytical approach becomes cumbersome. This is because the electric fields of the eigenmodes and/or reflection coefficients require lengthy calculations. In this section we describe a numerical method which allows us to simulate the emission from an anisotropic thin film stack in an arbitrary direction [19].

The simulation is based on a plane wave expansion of the dipole field in an infinite homogeneous anisotropic medium. The plane waves are then modulated by the wide angle and multiple beam interferences that arise from reflections onto the one-dimensional CLC stack. The reflection of plane waves with given $\mathbf{k}$-vector and arbitrary polarization on the CLC stack is described by a matrix method [22]. First the power emitted by a unit dipole $P(\lambda, \alpha)$ in a given CLC stack is calculated for each angle and wavelength separately. The spectral density at 


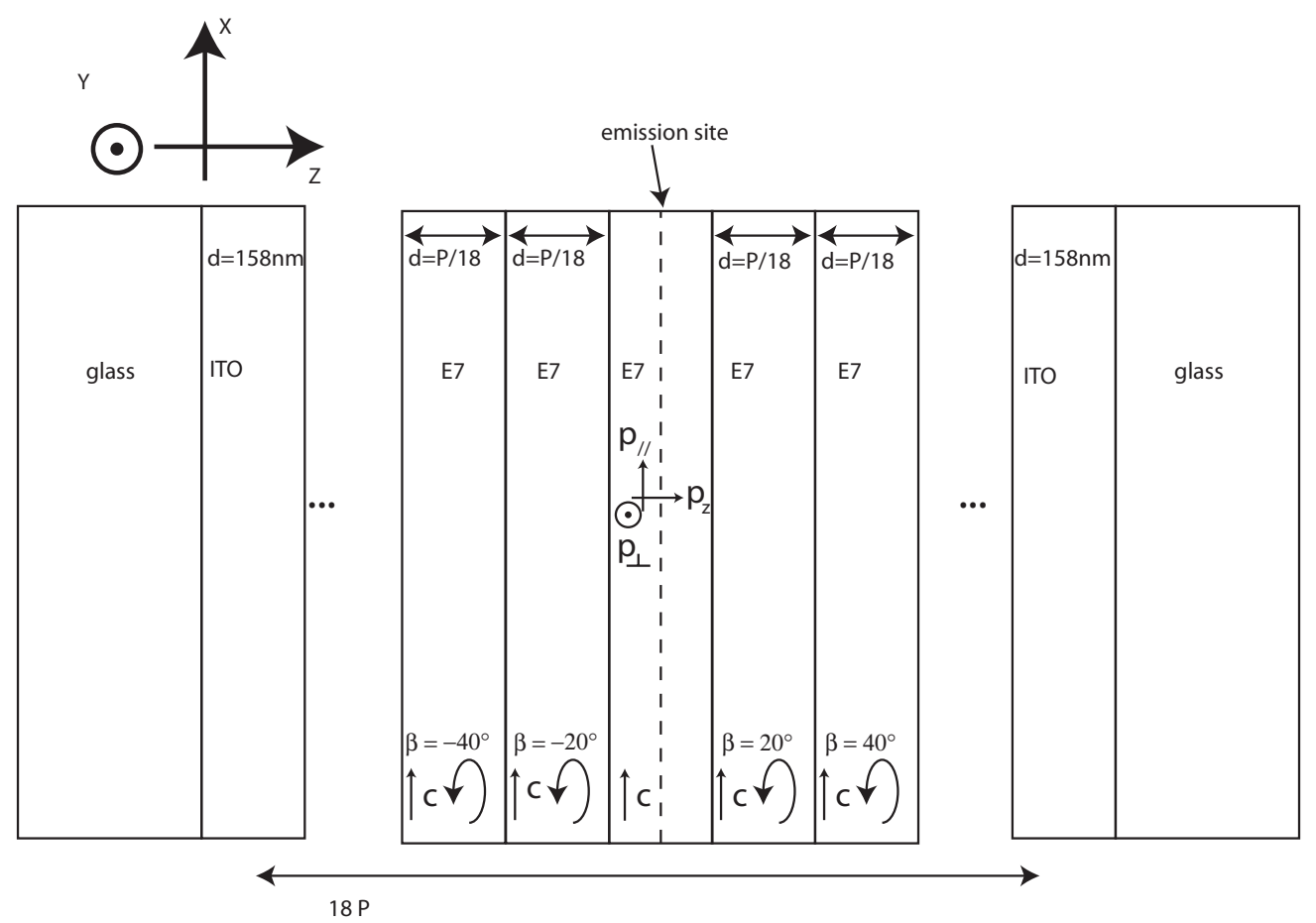

FIG. 3. Layer stack that approximates the continuous twist of a CLC between two substrates. Each pitch is divided into 18 homogeneous slabs with different orientations of the optical axis. Three orientations for the dipole transition are considered for simulation.

an angle $\alpha$ for a given CLC stack and dye $S_{\mathrm{CLC}}(\lambda, \alpha)$ is then obtained by weighing the emitted power with the normalized spectral density $S_{0}(\lambda)$ of the dye in E7, which is obtained by measuring the emission of a cell filled with a nonchiral mixture:

$$
S_{\mathrm{CLC}}(\lambda, \alpha)=S_{0}(\lambda) P(\lambda, \alpha) .
$$

The described algorithm has been implemented in a numerical simulation program to obtain the simulation results described in the following sections. The cell is treated as a series of discrete slabs; each slab is characterized by its thickness, the refractive indices, and the orientation of the optic axis (see Fig. 3). For the simulations in this paper, we use 18 slabs per pitch of the CLC, so the optical axis in each slab is rotated over $20^{\circ}$ compared to the previous slab. The entire CLC thickness is assumed to be 18 pitches long. The two refractive indices of the CLC mixture are assumed to be equal to those of undoped E7 $\left(n_{o, \mathrm{E} 7}=1.53-1.51\right.$ and $n_{e, \mathrm{E} 7}=1.75-1.72$ for $\lambda=500-780 \mathrm{~nm})$, since only a small amount of dopant was added. In simulation $P=365 \mathrm{~nm}$ was used; for this pitch the simulated spectrum best matches the measured spectrum. The CLC region is bound by a $158-\mathrm{nm}$ ITO $\left(n_{\text {ITO }}=1.98-1.75\right.$ for $\lambda=500-780 \mathrm{~nm}$ ) layer and a thick glass substrate on each side (for simplicity we ignore the alignment layers), and the emission into the glass substrate is calculated.

Simulations are carried out for three orientations of the transition dipole moment: parallel to the local $\mathrm{LC}$ director $\mathbf{p}_{/ /}$, perpendicular to the local LC director and the $z$ axis $\mathbf{p}_{\perp}$, and parallel to the $z$ axis $\mathbf{p}_{z}$. The degree of molecular orientation of the dye along the LC director is expressed via the order parameter $S_{\text {dye }}$. For a distribution of dipoles with, respectively, $\# p_{/ /}, \# p_{\perp}$, and $\# p_{z}$ dipoles along the 3 direction, $S_{\text {dye }}$ is given by Eq. (8), where we assume that in a uniaxial medium there is a uniform distribution of dipoles in the plane perpendicular to the extraordinary axis $\left(\# p_{\perp}=\# p_{z}\right)$ and $\# p_{/ /} / \# p_{\perp}=\rho$ :

$$
S_{\text {dye }}=\frac{3<\cos ^{2} \theta>-1}{2}=\frac{3 \frac{\rho}{\rho+2}-1}{2} .
$$

$\theta$ is the angle between the dipole moment and the LC director. When the dipole moment is randomly oriented $S_{\text {dye }}=0$. When the dipole moment aligns perfectly parallel (or perpendicular) to the director, $S_{\text {dye }}$ becomes 1 (or $-1 / 2$ ). The order parameter is accounted for in simulations by taking a weighted average over the three dipole moments. The relative weight for the three dipoles is determined by measurement of the linear polarization ratio $I_{/ /} / I_{\perp}=\# p_{/ /} / \# p_{\perp}$ in the nonchiral mixture. The emission for a cell, homogeneously doped with dye molecules, is found by incoherently adding the powers emitted by a representative ensemble of dipoles, namely three orthogonal dipoles in each slab of the CLC.

\section{RESULTS AND DISCUSSION}

In this section we compare and discuss the simulations and experiments obtained with the methods described above. First we consider the polarization and spectrum emitted perpendicular to the substrate; afterwards the emission for other angles is analyzed.

First a transmission spectroscopy measurement of the CLC device has been performed to identify the dye absorption and PBG properties [Fig. 4(a)]. The transmission measurement was done using unpolarized light; the transmission drops to around $50 \%$ in the PBG (instead of $0 \%$ for right-handed circular polarized light). Figure 4(a) also shows the simulated 

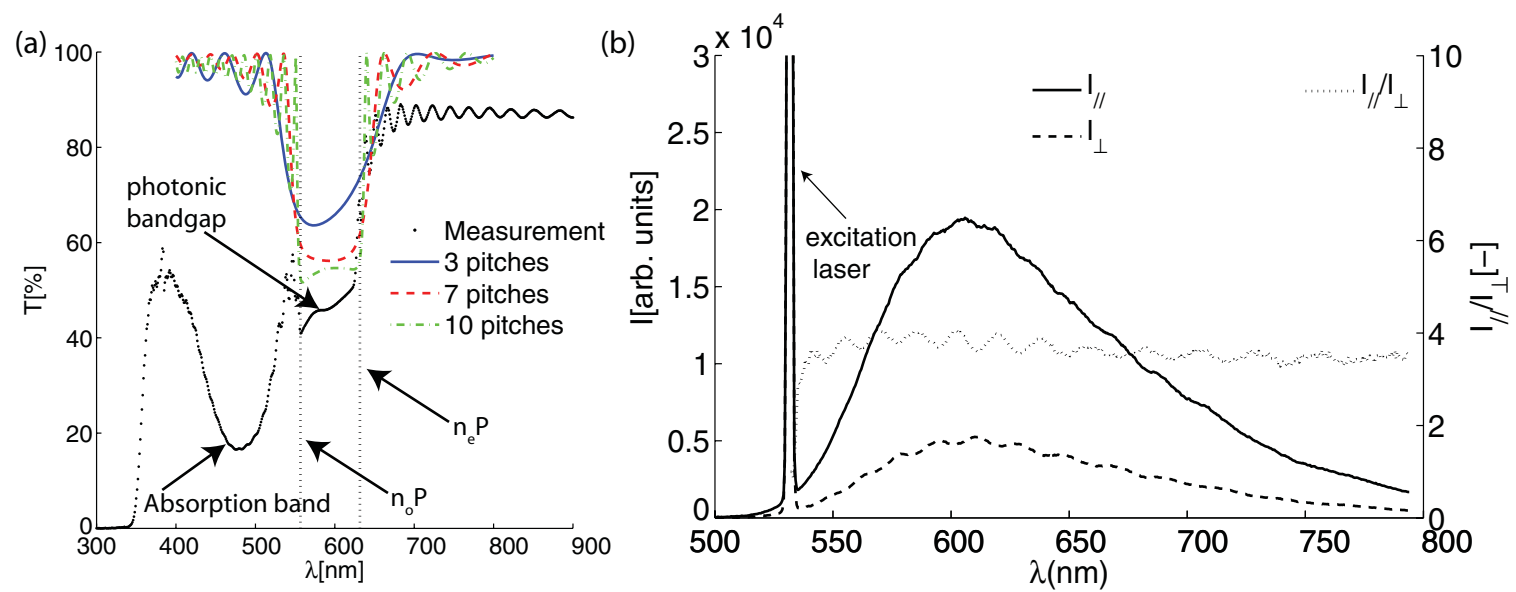

FIG. 4. (Color online) (a) Transmission spectrum of CLC mixture in cell. (b) Emission spectra with polarization parallel and orthogonal to the director for DCM in E7 with homogeneous alignment.

transmission (for unpolarized light) for different CLC thicknesses. In simulation the transmission also decreases to $50 \%$ as the thickness of the CLC increases. The absorption of the dye-doped CLC and reflection from the glass-air interfaces was neglected in the simulation. Figure 4(b) shows the measured fluorescence spectrum of the dye in E7 with homogeneous planar alignment for two different polarizations. $I_{/ /} / I_{\perp}$ is obtained from the ratio of the emissions for parallel and perpendicular polarization for a nonchiral mixture with the director parallel to the alignment direction. For DCM in E7 the ratio is only weakly dependent on the wavelength and $I_{/ /} / I_{\perp}=$ 3.7. This corresponds to an order parameter $S_{\text {dye }}=0.47$.

\section{A. Polarization and spectrum in the normal direction}

Figure 5 displays the fluorescent emission from the CLC mixture in the normal direction. Figure 5(a) compares the simulated spectrum for dipoles oriented completely parallel (green dash-dotted curve), perpendicular (red dashed curve), and randomly (blue solid curve). It is seen that a dipole parallel to the LC couples predominantly to the peak on the long wavelength side of the PBG. For dipoles perpendicular to the LC coupling to the short wavelength side is favored. Randomly oriented dipoles couple to both peaks. In Fig. 5(b) the measured spectrum is compared to the simulation for a random dipole (blue solid curve) and dipoles with order parameter $S_{\text {dye }}=$ 0.47 (red dashed curve). The previously measured value $S_{\text {dye }}=0.47$ yields a spectrum that more accurately matches the measured emission spectrum, in particular the peaks on the left- and right-hand sides of the PBG.

The peak locations given by the approximate formula Eq. (6) are compared to the measurement and simulation in the closeup in Fig. 5(b). Equation (6) slightly overestimates the wavelength of the emission peaks. This deviation is due to the fact that the exact minimum of $\left|1-r^{2}\right|$ is near (but not exactly at) the wavelength where $\arg \left(r^{2}\right)=0$ [the
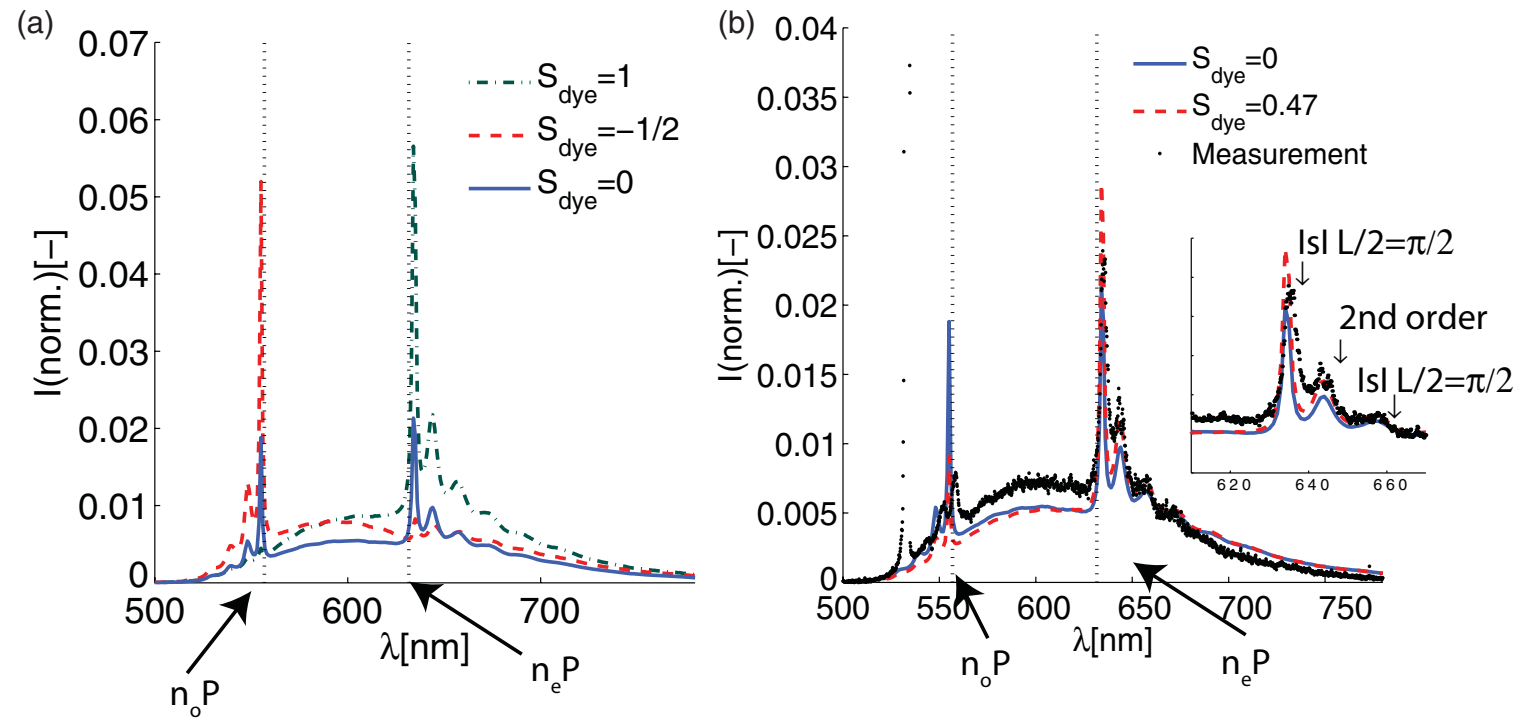

FIG. 5. (Color online) (a) Simulation of emission in the normal direction for dipoles: parallel, perpendicular, and random to LC ( $S_{\text {dye }}=$ $1,-1 / 2,0$ ). (b) Measured emission spectrum vs simulation. Simulation using the measured $S_{\text {dye }}=0.47$ matches the measured peak heights. Closeup: peak wavelengths using Eq. (6). 


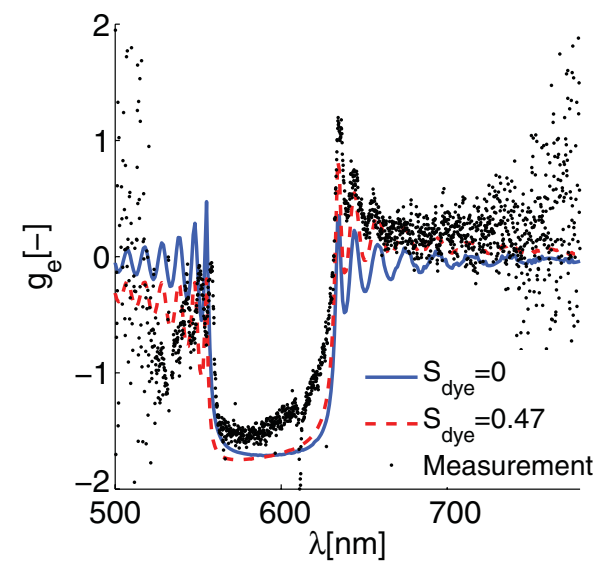

FIG. 6. (Color online) Measured and simulated dissymmetry factor $g_{e} \cdot g_{e}=-2,2$ correspond to circularly polarized light (respectively, with left- and right-handedness). Inside the PBG only the allowed circular polarization is emitted. Outside of the PBG, the emission of the other polarization is enhanced and $g_{e}$ changes rapidly as a function of the wavelength. approximation made to derive Eq. (6)]. The second order peak is found by applying the method detailed in Sec. III A for a dipole at three quarters of the CLC thickness. The electric field of the second order peak is zero in the middle of the CLC, hence a dipole in the middle of the CLC does not couple to it.

The degree of circular polarization is expressed as the dissymmetry factor $g_{e}$ :

$$
g_{e}=2 \frac{I_{l}-I_{r}}{I_{l}+I_{r}} .
$$

$g_{e}=-2$ and $g_{e}=2$ correspond to completely circularly polarized light, respectively, with left- and right-handedness. Figure 6 plots the measured and simulated $g_{e}$. Inside the PBG only circular polarization with left-handedness is emitted and $g_{e}$ is close to -2 . The dipoles at the borders of the cell only experience a PBG on one side and therefore contribute also to the other circular polarization. At the edges of the PBG the emission of circular polarized light with right-handedness is strongly enhanced; as a consequence the overall $g_{e}$ suddenly becomes positive. The absolute value of $g_{e}$ is not as high as inside the PBG region since all dipoles still emit into both circular polarization states.
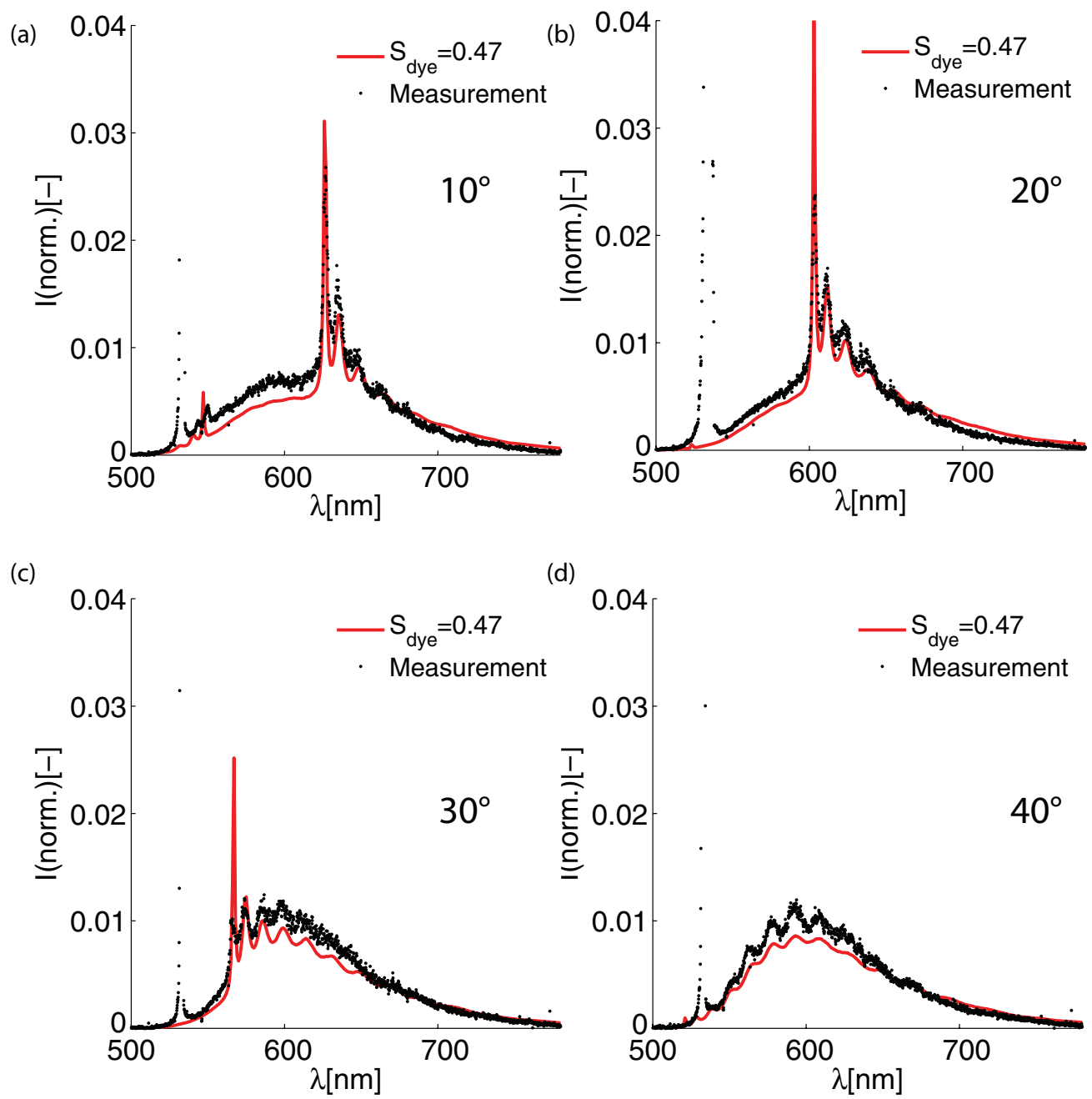

FIG. 7. (Color online) Angle dependency of emission spectrum $a$ of CLC cell. Comparison of experiments (dots) and simulation (solid lines). (a), (b), (c), and (d) show measurements at angles of $10^{\circ}, 20^{\circ}, 30^{\circ}$, and $40^{\circ}$ in glass, respectively. 
Again the simulation which takes into account the order parameter (red dashed line) better matches the measured spectral dependency of $g_{e}$, in particular the relative height of the peaks at the right- and left-hand side of the PBG. For a randomly oriented dipole both peaks are equally excited and the polarization is symmetric around the center of the PBG. For an emitter oriented along the LC director, the long wavelength peak is more strongly excited and the long wavelength peak becomes more polarized.

\section{B. Emission into oblique angles}

When a CLC is observed at more oblique angles, the PBG shifts towards shorter wavelengths. This is because the condition that the phase of the electric field changes by $\pi$ over one period (for the central wavelength $\lambda_{c}$ of the PBG) is fulfilled for shorter wavelengths as the inclination angle $\alpha_{\mathrm{CLC}}$ in the CLC ( $\left.\alpha_{\text {CLC }} \approx \alpha_{\text {glass }}\right)$ increases:

$$
\frac{2 \pi}{\lambda_{c}} \sqrt{\varepsilon_{\text {avg }}} \cos \left(\alpha_{\mathrm{CLC}}\right)=\frac{2 \pi}{P} .
$$

The shift of the PBG to shorter wavelengths is apparent in the emission spectrum of a CLC at higher angles (Figs. 7(a), 7(b), $7(\mathrm{c})$, and 7 (d) show the emission spectrum at $10^{\circ}, 20^{\circ}, 30^{\circ}$ and $40^{\circ}$, respectively). As the angle increases the PBG and peaks in the emission spectrum shift towards shorter wavelengths. At an angle of $20^{\circ}$ the left edge of the PBG is smaller than the excitation wavelength and this emission peak disappears. For even larger angles the entire PBG no longer overlaps with the emission spectrum of the dye and only a small modulation of the intrinsic spectrum $S_{0}$ is observed. The shift of the emission spectrum is accurately reproduced by the simulations (red solid curves).
For large angles $\left(40^{\circ}\right)$ the measured spectrum shows a modulation with the wavelength, which is also visible, but less pronounced in the simulation. This is caused by interference of reflections between the ITO boundaries. The period of the fringes is the same in measurement and simulation and is related to the thickness of the cell.

\section{CONCLUSIONS}

The fluorescence of a dye is significantly modified by doping it into a cholesteric liquid crystal. Emission is suppressed inside the stop band and enhanced near the band edge. A numerical method based on a plane wave expansion is used to model emission for normal and oblique angles. The same method is also applicable to more complex layer stacks. In previous models only propagation under oblique angles through CLC or normal emission was treated. Furthermore the presented method does not require knowledge of the eigenmodes of propagation and can be applied to very general configurations. The results of the numerical model are in good agreement with experimental results for the polarization and spectral properties of the emission. This numerical model is expected to facilitate the design of light emitting devices and dye lasers using liquid crystals.

\section{ACKNOWLEDGMENTS}

The work leading to these results has received funding from the IWT (Flemish Institute for Science and Technology) and the European Community's Seventh Framework Programme (PITN-GA-2008-215399-FINELUMEN). The authors acknowledge the Belgian Science Policy (Project IAP 6/10-photonics@be).
[1] K. Driesen, D. Moors, J. Beeckman, K. Neyts, C. GorllerWalrand, and K. Binnemans, J. Lumin. 127, 611 (2007).

[2] W. De Cort, J. Beeckman, R. James, F. A. Fernandez, R. Baets, and K. Neyts, Opt. Lett. 34, 2054 (2009).

[3] G. Overton, Laser Focus World 45, 44 (2009).

[4] T. H. Lin, H. C. Jau, C. H. Chen, Y. J. Chen, T. H. Wei, C. W. Chen, and A. Y. G. Fuh, Appl. Phys. Lett. 88, 3 (2006).

[5] H. Finkelmann, S. T. Kim, A. Munoz, P. Palffy-Muhoray, and B. Taheri, Adv. Mater. 13, 1069 (2001).

[6] K. Funamoto, M. Ozaki, and K. Yoshino, Jpn. J. Appl. Phys., Part 2 42, L1523 (2003).

[7] H. Coles and S. Morris, Nat. Photonics 4, 676 (2010).

[8] A. D. Ford, S. M. Morris, and H. J. Coles, Mater. Today 9, 36 (2006).

[9] H. de Vries, Acta Crystallogr. 4, 219 (1951).

[10] E. I. Kats, Sov. Phys. JETP 32, 1004 (1971).

[11] C. Oldano, Phys. Rev. A 31, 1014 (1985).

[12] A. Lakhtakia and W. S. Weiglhofer, Microwave Opt. Technol. Lett. 12, 245 (1996).
[13] H. Wohler, G. Haas, M. Fritsch, and D. A. Mlynski, J. Opt. Soc. Am. A 5, 1554 (1988).

[14] D. W. Berreman, J. Opt. Soc. Am. 62, 502 (1972).

[15] P. Pollmann, K. J. Mainusch, and H. Stegemeyer, Z. Phys. Chem. (Frankfurt) 103, 295 (1976).

[16] M. Voigt, M. Chambers, and M. Grell, Chem. Phys. Lett. 347, 173 (2001).

[17] J. Schmidtke and W. Stille, Eur. Phys. J. B 31, 179 (2003).

[18] T. Matsui and M. Kitaguchi, Appl. Phys. Express 3, 061701 (2010).

[19] L. Penninck, P. De Visschere, J. Beeckman, and K. Neyts, Opt. Express 19, 18558 (2011).

[20] C. Oldano, E. Miraldi, and P. T. Valabrega, Phys. Rev. A 27, 3291 (1983).

[21] P. Yeh and C. Gu, Optics of Liquid Crystal Displays, Wiley Series in Pure and Applied Optics (Wiley, New York, 1999), pp. xii, 438, 282-305.

[22] D. Y. K. Ko and J. R. Sambles, J. Opt. Soc. Am. A 5, 1863 (1988). 